\title{
Activity and rest alternation: temporal distribution and influencing factors in nocturnal rodents
}

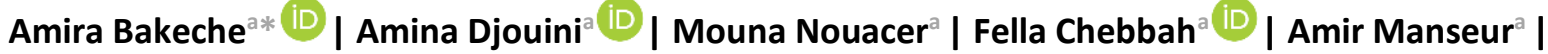 \\ Chahira Retem $^{\text {a }}$ Abdelmadjid Bairi ${ }^{\mathrm{i}}$ (D) | Abdelkrim Tahraoui ${ }^{\mathrm{a}}$
}

applied Neuroendocrinology Laboratory, Department of Biology, Faculty of Sciences, University Badji Mokhtar, Annaba, Algeria.

Corresponding author: bakecheAmira@outlook.fr

\begin{abstract}
Lifestyle and heredity are two different terms but yet closely related components. An evaluation for the time invested in sleep and locomotion behaviors per second in the Wistar rats animal model was carried out. A total of six rats within the same cage were marked by color in a room supported by the day's light (12h lighting/12h darkness). These animals were filmed for $18 \mathrm{~h}$ daily for ten days (9h lighting, 9h darkness) to estimate the time budget of sleep and locomotion behaviors and the temporal distribution taking into account the nature of this biological model's activity. The results obtained reveal sleeping/locomotion cycles respecting the natural photoperiod except for a rat that shows dominance in nocturnal sleep compared to the rest of the rats. These advances support a change in photoperiodic behavior in response to an adaptation to the rhythm imposed by the manipulator. They invite studies on a larger sample to consolidate this behavior controlled by the environment.
\end{abstract}

Keywords behavior, environment, photoperiod, rat, time use

\section{Introduction}

Besides the free-living rodent populations, various types of Norway rats ("Wistar-type" or "hooded-type") are extensively used as experimental animal models for diverse scientific research projects (Baker et al 2013; Boros et al 2019). Wistars, selected by Donaldson at the Wistar Institute (USA) in 1906, is a versatile inbred strain used in all disciplines of medical and biological researches (Iliuţã 2011), which is active at night; their main sleep phase occupies the day (Kronfeld-schor and Dayan 2008; Oosthuizen 2020). In rodents, as in all animal species, the alternation of light and darkness is the primary synchronizer of circadian rhythms (Benstaali et al 2001; Chen et al 2019). The frequency of activity and rest is generated by two neural groups located on the hypothalamus floor, the suprachiasmatic nuclei (NSC). Their destruction removes the activity-rest cycle; their transplantation restores this rhythm (Reppert and Moore 1991; Mieda 2019).

The rats' handling and interview procedures during their resting phase cause stress that, even in the short term, may affect their well-being (Abou-ismail et al 2008). The precise point in the circadian cycle to which these practices are applied is therefore essential; this moment may result in additive stress due to the disruption of the wake-sleep cycle in this nocturnal species. Koch et al (2017) emphasize the importance of a day as a factor that can shape an animal's response to stress.

In this context, knowledge of each biological model's phase response curve is ideal for defining an experimental protocol's optimal moment. Thus, the present study aims to assess the distribution of nocturnal investment time behavior of sleep, necessary steps to daily repetition and locomotion, and essential faculty to move in Wistar rats.

\section{Materials and Methods}

\subsection{Animal housing and livestock}

The experiment was carried out using a batch of six rats (Pasteur Institut Algiers, Algeria) following 1 of the 3Rs of the foundation of the ethical approach in animal experimentation: to reduce, refine and replace (Russell and Burch 1959). The rats had between nine to ten weeks old grouped in a single isolated cage in a box at the animal house to avoid disruptive factors. Rats were housed randomly in a standard polyethylene cage $(48.5 \mathrm{~cm}$ long, $33 \mathrm{~cm}$ wide, and $21 \mathrm{~cm}$ high). The cage was supported with sawdust as bedding material, and feed and water were provided ad libitum. The rats were kept under a $12 \mathrm{~h}$ lighting and $12 \mathrm{~h}$ darkness photoperiod. The ambient temperature was adjusted at (between 19 and $23^{\circ} \mathrm{C}$ ). The rats were marked from zero $(0)$ to five $(5)$, ensuring that the numbers remain visible.

\subsection{Experimental monitoring}

In the absence of any treatment, the rats were observed in natural photoperiod at $18 \mathrm{~h}$ per day ( $9 \mathrm{~h}$ in the daytime and $9 \mathrm{~h}$ at night) for ten days. The rats were weighed daily for ten days before and after each observation using an electronic digital scale. The cage was cleaned twice a day before and immediately after each follow-up, detergent-free; the rats were removed from their cage and placed in a clean cage with clean bedding. The tracking was saved using a 
camera with an infrared filter (dark phase observation), armed with two memory cards with a capacity of 64GB each to record $18 \mathrm{~h}$. The time invested in each of sleep and activity behaviors was examined.

\subsection{Behavioral data}

The sampling of behaviors taken into account was always carried out for each behavior by two observers, who were introduced into the experimental room 40 min before the scheduled start of observation to allow the rats to adapt their presence, weigh them, clean the cage and start the camera. Sleep and locomotion behaviors like climbing, exploration, social interaction, and immobility were followed in rats placed in a room lit during the daytime, daylight, and dark in the night phase. The whole approach described is in agreement with the University of Badji Mokhtar - Annaba's Ethics guide.

\subsection{Statistics}

The daily time-use in seconds for each rat of each of the two behaviors studied was treated with the XLSTATPRO

(S) $\quad$ Sleep $\quad$ Locomotion

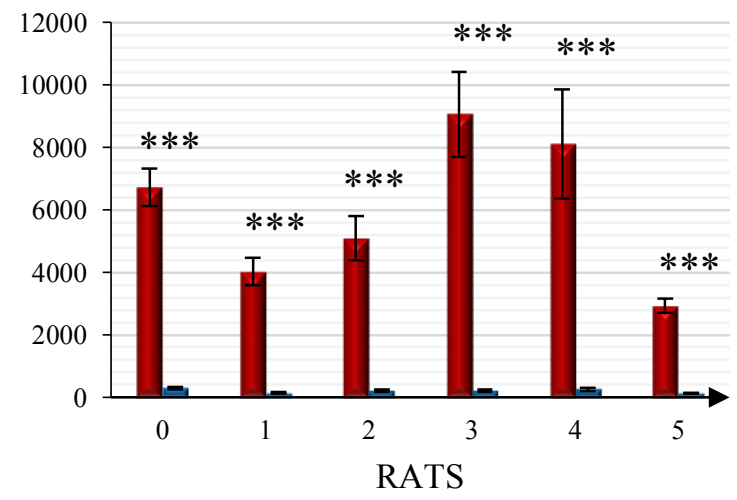

Figure 1 Sleep and locomotor behaviors during 10 days of observation in diurnal phase (Mean $\pm S E, n=6$ ). Not significant difference $P>0.05 ; P^{*} \leq 0.05 ; P^{* *} \leq 0.01 ; P^{* * *} \leq 0.001$.

Second day

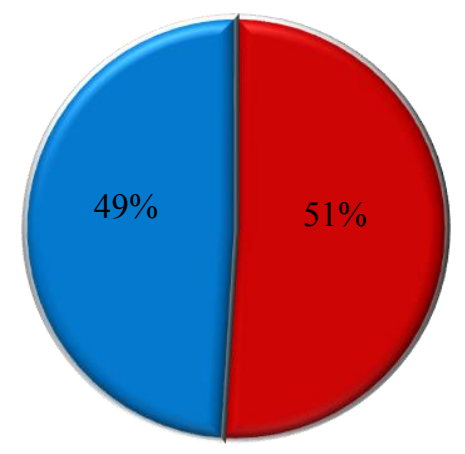

回Sleep 回Locomotion software version 2016. The data represented on average \pm standard deviation of mean and compared by a Student test according to the ratio $P$-value calculated / at ALPHA significance level $=0.05$.

\section{Results}

Changes in sleep time and locomotion during the daytime phase in the six rats during the 10-day observation indicated that sleep time was greater than that of movement for the entire group with a variable time-budget (Figure 1).

Changes in sleep time and locomotion during the nocturnal phase in the six rats over a 10-day observation period showed that sleep time is less than that of movement in the group as a whole. Rat 1 is distinguished by investing a higher time in the two observed behaviors than its congeners (Figure 2).

We recorded in detail the time invested in sleep and locomotion in rats 1 to 2 nd and 8th day of observation with $51 \%$ at day 2, 59\% at day 8 in rest, and $49 \%$ at day 2, $41 \%$ at day 8 in locomotion (Figure 3 ).

\section{(S) $\quad$ Sleep $\quad$ Locomotion}

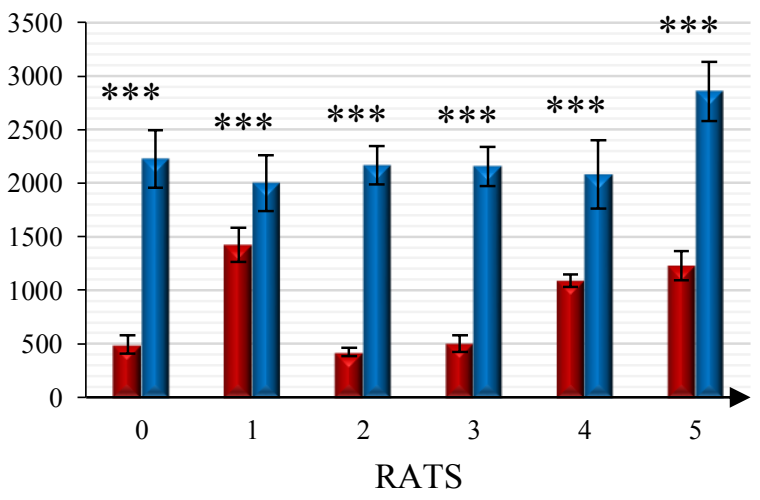

Figure 2 Sleep and locomotor behaviors during 10 days of observation in nocturnal phase (Mean $\pm \mathrm{SE}, \mathrm{n}=6$ ). Not significant difference $P>0.05 ; P^{*} \leq 0.05 ; P^{* *} \leq 0.01 ; P^{* * *} \leq 0.001$.

Eighth day

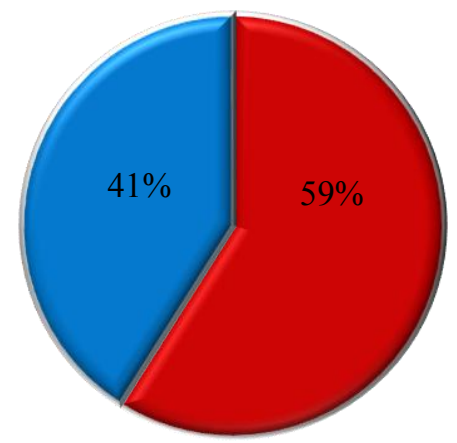

回Sleep 國Locomotion

Figure 3 Sectors of sleep and locomotor behaviors in rat- 1 in the nocturnal phase on the $2^{\text {nd }}$ and $8^{\text {th }}$ days of observation. 


\section{Discussion}

The rotation of the earth around its axis generates a light-dark alternation whose period is 24 hours. Throughout the evolutionary process, living organisms have developed an internal biological clock that allows them to adapt to cyclical changes in the external environment, orchestrating their physiological and behavioral processes over time (Halberg 1963; Boyenval 2017; Abbott and Zee 2019).

The alternation of activity and rest during the 24 hours or activity-rest cycle was observed in animal species, as diverse as the Drosophila, rat, mouse, hamster, and man. First called "nycthemeral", the activity-rest cycle is considered a circadian rhythm because of its relationship with day and night alternation. It persists under constant environmental conditions in all species mentioned above (Reppert and Moore 1991; Mieda 2019).

Today, it is known that NSC distributes the circadian signal through humoral, nervous, hormonal, and behavioral pathways (Pevet and Challet 2011). In-vivo electrophysiology in rats demonstrated that NSC neurons exhibit rhythmic electrical activity with a higher activity level during the day (Inouye and Kawamura 1979; Tso et al 2017).

Sleep occupies the most crucial place in the rat's time budget as they spent more than $60 \%$ of their time sleeping (Hurst et al 1996; Barker et al 2017). Alfoldi et al (1990) and Frank et al (2017) reported that in nocturnal rodents such as rats, sleep occupies $80 \%$ of the daylight and $20 \%$ dark time.

The $10^{\text {th }}$-day follow-up report included 18 hours of distribution throughout the day and night in the rats' group, reinforcing this rodent's mainly diurnal nature. Except for rat1 , the sources mentioned above have a longer sleep time, unlike these congeners. Therefore, a non-normal activity time, especially on the second and eighth days, required a record sleep investment with nearly $60 \%$ of the overall observation time.

From there a probability, this rat borrows the path of a phenotypic modification which can disguise its answer in various aversive conditions or not. This difference would undoubtedly be rooted in manipulation by the researcher confounding the active phase and resting mammals. Even if this result reported a form of adaptation of the idle activity cycle imposed by the manipulator, it would not be inconsequential as Abou-ismail et al (2008) showed that cage hygiene and the rat's care could have an impact on his wellbeing.

Achieved during the day, that is, during the sleep time, these care behaviors contributed to the rat development of malaise signs (enlarged thymus size, more frequent aggressive behavior, chromodacryorrhea, and decreased frequency of grooming behavior). Therefore, it is advisable to perform this care during the rat's activity time and let him sleep when he needs it.

In its natural environment, the activity of the rat is somewhat nocturnal or twilight. It sleeps instead during the day and is active at dawn, evening, and night. They spend much time exploring their cage and jumping on all the surfaces they have. It is not habitual for them to spend much time burrowing under their litter or trying to dig the cage's floor (Sirois 2015).

Many behaviors are common to all rodent species. In captivity and precisely in rats, we note the locomotor behavior burial under the litter or foral exploration of the cage, jump, and climbing the cage grid. These behaviors are accompanied by a phase of immobility well installed in the nit made of litter and occupying one from the four corners of the cage before falling asleep with repeated episodes and mainly at the end of the night (Djouini et al 2017). From acrobatics to confrontation, social interactions are also a form of mobility expressed by these mammals, if necessary.

The results recorded a time of activity faithful to that of any higher rodent at night compared to the day. Like sleep, this behavior differs in rats- 1 if one compares it with the rest of the rats. It has times that are close to or close to those of sleep over the ten days. On the second and eighth day, we observed a regression of the locomotion time. This one takes a value lower than that of the sleep in period supposed to be of activity with values of $49 \%$ respectively $(1232 \pm 363$ seconds) and $41 \%$ or ( $861 \pm 333$ seconds) of global tracking time.

\section{Conclusions}

This follow-up revealed a heterogeneous pattern and suggested a reversal hypothesis of the nycthemeral behavior for the rat. This inversion, probably in response to a rhythm imposed by the manipulator, involves environmental or even genetic factors that can lead this rat to adapt to daytime liveliness.

\section{Conflict of Interest}

The authors declare that there are no conflict of interest with this work.

\section{Funding}

This research did not receive any financial support.

\section{References}

Abbott SM, Zee PC (2019) Circadian Rhythms: Implications for Health and Disease. Neurologic clinics 37:601-613.

Abou-ismail UA, Burman OHP, Nicol CJ, Mendl M (2008) Let sleeping rats lie: does the timing of husbandry procedures affect laboratory rat behaviour, physiology and welfare?. Applied Animal Behaviour Science 111:329-341.

Alfoldi P, Tobler I, Borbely AA (1990) Sleep regulation in rats during early development. American Journal of Physiology 258:R634-R644.

Baker HJ, Lindsey J, Wesibroth SH (2013) The laboratory rat: biology and diseases. Elsevier, Volume 1.

Barker TH, George RP, Howarth GS, Whittaker AL (2017) Assessment of housing density, space allocation and social hierarchy of laboratory rats on behavioural measures of welfare. Plos One 12:e0185135.

Benstaali C, Mailloux A, Bogdan A, Auzeby A, Touitou Y (2001) Circadian rhythms of body temperature and motor activity in rodents: their relationships with the light-dark cycle. Life Sciences 68:2645-2656.

Boros Á, Orlovácz, K, Pankovics P, Szekeresb S, Földvárib CG, Fahsbenderd E, Delwartd, EE, Reuter G (2019) Diverse picornaviruses are prevalent among free-living and laboratory rats (Rattus norvegicus) in Hungary and can cause 
disseminated infections. Infection Genetics and Evolution. 75:103988.

Boyenval D (2017) Étude et modélisation qualitative d'une voie de synchronisation des horloges circadiennes périphériques par le noyau suprachiasmatique.

Chen X, Liu CN, Fenyk-melody JE (2019) Effects of Sodium Lighting on Circadian Rhythms in Rats. Journal of the American Association of Laboratory Animal 58:311.

Djouini A, Haloui M, Bakeche A, Boutefnouchet I, Bairi A, Tahraoui A (2017) Approche quantitative des comportements nocturnes chez le rat wistar. Journal of Animal and Plant Sciences 34:5482-5490.

Frank MG, Ruby NF, Heller HC, Franken P (2017) Development of circadian sleep regulation in the rat: a longitudinal study under constant conditions. Sleep 40.

Halberg F (1963) Circadian (about twenty-four-hour) rhythms in experimental medicine [Abridged], 253-257.

Hurst JL, Barnard CJ, Hare R, Wheeldon EB, West CD (1996) Housing and welfare in laboratory rats: time-budgeting and pathophysiology in single-sex groups. Animal Behaviour 52:335-360.

Iliuţã A (2011) Experimental use of animals in research. Balneo-Research Journal 2:1-5.

Inouye SIT, Kawamura H (1979) Persistence of circadian rhythmicity in a mammalian hypothalamic" island" containing the suprachiasmatic nucleus. Proceedings of the National Academy of Sciences 76:5962-5966.
Koch CE, Leinweber B, Drengberg BC, Blaum C, Oster H (2017) Interaction between circadian rhythms and stress. Neurobiology of stress 6:57-67.

Kronfeld-schor N, Dayan T (2008). Activity patterns of rodents: the physiological ecology of biological rhythms. Biological Rhythm Research 39:193-211.

Mieda M (2019) The network mechanism of the central circadian pacemaker of the SCN: do AVP neurons play a more critical role than expected? Frontiers in neuroscience 13:139.

Oosthuizen MK (2020) Temporal flexibility in activity rhythms of a diurnal rodent, the ice rat (Otomys sloggetti). Chronobiology International 37:824835.

Pevet P, Challet E (2011) Melatonin: both master clock output and internal time-giver in the circadian clocks network. The Journal of Physiology. 105:170-182

Reppert S, Moore RY (1991) Suprachiasmatic nucleus: the mind's clock. Oxford University Press, USA.

Russell WMS, Burch RL (1959) The Principles of Humane Experimental Technique. London, UK: Methuen, xiv +: 238.

Sirois M (2015) Laboratory Animal and Exotic Pet Medicine-E-Book: Principles and Procedures. Elsevier Health Sciences.

Tso CF, Simon T, Greenlaw AC, Puri T, Mieda M, Herzog ED (2017) Astrocytes regulate daily rhythms in the suprachiasmatic nucleus and behavior. Current Biology 27:1055-1061. 